\title{
A Fundamental 90-GHz CMOS VCO Using New Ring-Coupled Quad
}

\author{
Zuo-Min Tsai, Member, IEEE, Chin-Shen Lin, Student Member, IEEE, C. F. Huang, John G. J. Chern, and \\ Huei Wang, Fellow, IEEE
}

\begin{abstract}
A new circuit topology, named ring-coupled quad for millimeter-wave voltage controlled oscillator (VCO) design, is proposed. The proposed circuit topology provides higher open loop voltage gain than conventional cross-coupled pair. The layout of the proposed ring-coupled quad is fully symmetric without additional interconnection lines. A 90-GHz VCO using 90-nm CMOS process is implemented with this ring-coupled quad. This $90-\mathbf{G H z}$ oscillator demonstrates a $2.5-\mathrm{GHz}$ tuning range and higher than $-20 \mathrm{dBm}$ output power. The proposed ring-coupled quad is suitable for the realization of high frequency VCOs.
\end{abstract}

Index Terms-Cross-coupled, voltage controlled oscillator (VCO), millimeter-wave.

\section{INTRODUCTION}

$\mathbf{V}$ OLTAGE controlled oscillators (VCOs) are key components for wireless communication systems. Because of the rapid promotion of CMOS technology, it is possible to realize CMOS VCOs for millimeter-wave applications. The push-push topology is used to generate the millimeter wave signal using the second harmonic signal of VCOs [1]-[4]. Due to low device gain and high transmission line loss, the fundamental VCO is not easy to realize at millimeter-wave frequency. A four-stages amplifier to increase the open loop gain [5] and a special layout for cross-coupled pair to improve the loss of the interconnections and the circuit symmetry [6] have been reported.

In this letter, a new circuit topology, named ring-coupled quad, is proposed. This ring-coupled quad uses the four-stage common source transistors so that the voltage open loop gain is higher than conventional cross-coupled pair. Moreover, the layout of the ring-coupled quad can be fully symmetric and the loss of the interconnection is significantly reduced. A $90-\mathrm{GHz}$ VCO using the proposed ring-coupled quad in a 90-nm CMOS process is implemented. The tuning range is $2.5 \mathrm{GHz}$ and the output power is higher than $-20 \mathrm{dBm}$. The experimental results show that the proposed ring-coupled quad is suitable for high frequency VCOs.

Manuscript received October 17, 2006; revised November 3, 2006. This work was supported in part by TSMC-NTU JPD and the National Science Council of Taiwan, R.O.C. under Projects NSC 94-2752-E-002-003-PAE, NSC 94-2219-E002-006, NSC 94-2219-E-002-009, and NSC 94-2213-E-002-008 and by the National Chip Implementation Center of Taiwan.

Z.-M. Tsai, C.-S. Lin, and H. Wang are with the Graduate Institute of Communication Engineering and the Department of Electrical Engineering, National Taiwan University, Taipei 100, Taiwan, R.O.C. (e-mail: hueiwang@ew.ee.ntu. edu.tw).

C. F. Huang and J. G. J. Chern are with the Taiwan Semiconductor Manufacturing Company, Ltd. (TSMC), Hsinchu 300, Taiwan, R.O.C.

Color versions of one or more of the figures in this letter are available online at http://ieeexplore.ieee.org.

Digital Object Identifier 10.1109/LMWC.2006.890499

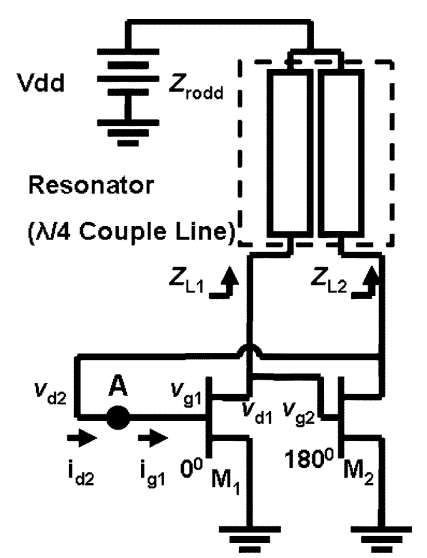

(a)

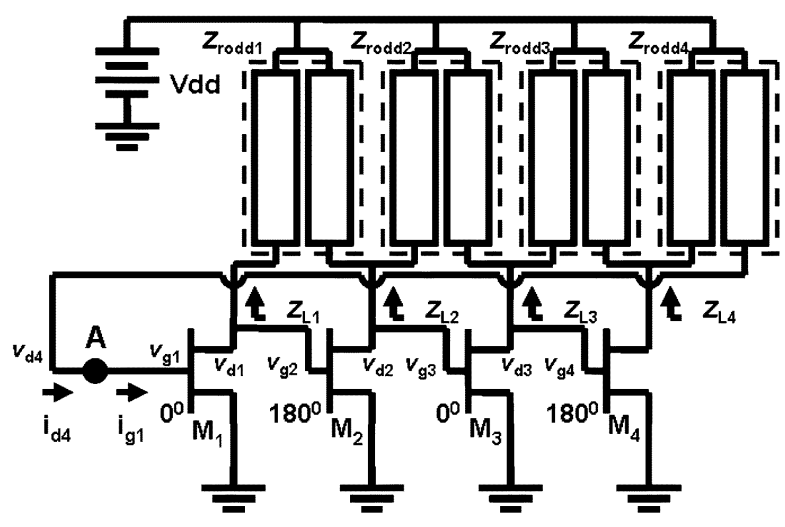

(b)

Fig. 1. Circuit schematic of (a) cross-coupled oscillators and (b) ring-coupled oscillators.

\section{CONCEPT OF RING-COUPLED OSCILLATOR}

Fig. 1(a) illustrates the schematic diagram of a conventional cross-coupled oscillator. It is composed of two common-source transistors $\left(M_{1}\right.$ and $\left.M_{2}\right)$ and one resonator with load impedance $\left(Z_{L 1}\right.$ and $\left.Z_{L 2}\right)$. The open loop voltage gain from gate of $M_{1}$ to drain of $M_{2}$ can be expressed in terms of device transconductance $\left(g_{m 1}\right.$ and $\left.g_{m 2}\right)$ and $Z_{L}$

$$
v_{d 2} / v_{g 1}=\left(-Z_{L 1}(\omega) g_{m 1}\right)\left(-Z_{L 2}(\omega) g_{m 2}\right) .
$$

If the imaginary parts of $Z_{L 1}$ and $Z_{L 2}$ equal to zero, the phase of the open loop voltage gain is $0^{\circ}$ and the signal is out of phase between each stages. When the real part of $1 / Z_{L 1}$ and $1 / Z_{L 2}$ are lower than the value of $g_{m 1}$ and $g_{m 2}$, the magnitude of the open loop voltage gain is higher than unity and the oscillation condition is satisfied. Since the voltage of each stage is out-ofphase, the resonator can be realized as a pair of coupled lines and 


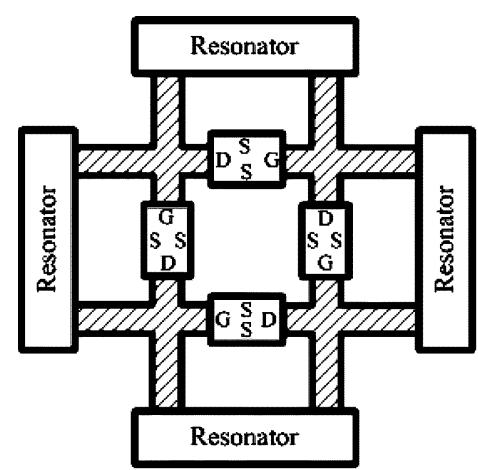

Fig. 2. Schematic of the VCO using ring-coupled quad.

the load impedance $\left(Z_{L 1}\right.$ and $\left.Z_{L 2}\right)$ are equal to the odd mode impedance of the coupled line $\left(Z_{\text {rodd }}\right)$.

Following the principle of a cross-coupled pair, the positive feedback can be realized with the four-stage common-source transistors. Fig. 1(b) illustrated the proposed ring-coupled oscillator. Four transistors are cascaded and four resonators are connected to the source and drain of each stage. The open loop voltage gain becomes

$$
\begin{aligned}
v_{d 4} / v_{g 1}=\left(-g_{m 1} Z_{L 1}(\omega)\right)\left(-g_{m 2} Z_{L 2}(\omega)\right) & \\
& \times\left(-g_{m 3} Z_{L 3}(\omega)\right)\left(-g_{m 4} Z_{L 4}(\omega)\right) .
\end{aligned}
$$

Similar to (1), the signal is out of phase at each stage. The phase of the open loop voltage gain is zero while the imaginary part of the load admittance $\left(1 / Z_{\mathrm{Li}}(\omega), i=1,2,3,4\right)$ is zero. Since the real part of the load admittances are lower then the transconductance of the transistors, the magnitude of the loop gain is higher than unity. Because of the open loop gain in (2) due to four-stage cascade transistors, the magnitude of the loop gain is higher than the traditional cross-coupled pair. At higher frequency, the margin of the load admittance for oscillation condition is less due to the substrate loss. Since there are four-stage transistors, if the loop gain of one stage is lower, the other stages can cover the loss and ensure the oscillation condition.

Another important feature of using this proposed ring-coupled quad in VCO design is the symmetric layout. In the conventional cross couple pair, a cross junction is required to connect the gate and the drain of each transistor. As illustrated in the ring-coupled quad (Fig. 2), the four-transistors are connected in a ring, so that no additional lines are added in the layout and the parasitics of the interconnections are significantly reduced.

\section{MMIC PROCESS AND THE 90-GHz VCO}

The process used in this VCO is TSMC 1P9M CMOS technology, with an $f_{T}$ of $160 \mathrm{GHz}$ and an $f_{\max }$ of $142 \mathrm{GHz}$. In order to reduce the loss of the transmission line in CMOS process, the thin film microstrip line is adopted [7]. There are nine metal layers in this 90-nm CMOS process. Metal 1 is used for the ground and the top metal is used for the signal line. A pair of coupled thin-film microstrip lines are used as the resonator which has a odd-mode characteristic impedance of $65.5 \Omega$ and odd-mode attenuation of $560 \mathrm{~dB} / \mathrm{m}$. Breaking point $\mathrm{A}$ in Fig. 1(a) and (b), the voltage or the current transfer function is simulated by the image parameter method [8]. Fig. 3 shows the simulated transfer functions of oscillators in

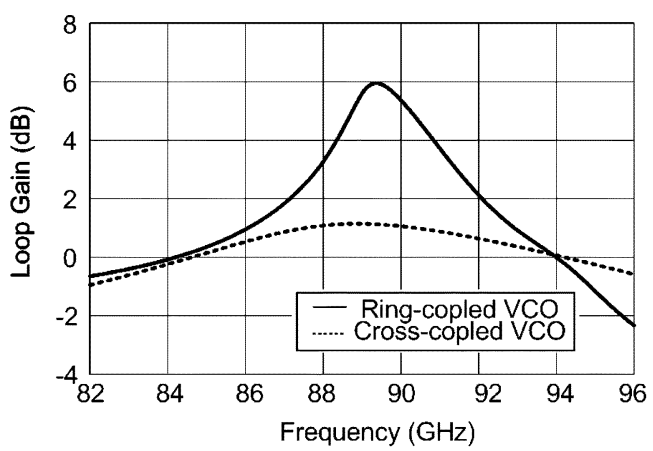

Fig. 3. Simulated loop gain of the proposed ring-coupled VCO and the crosscoupled VCO

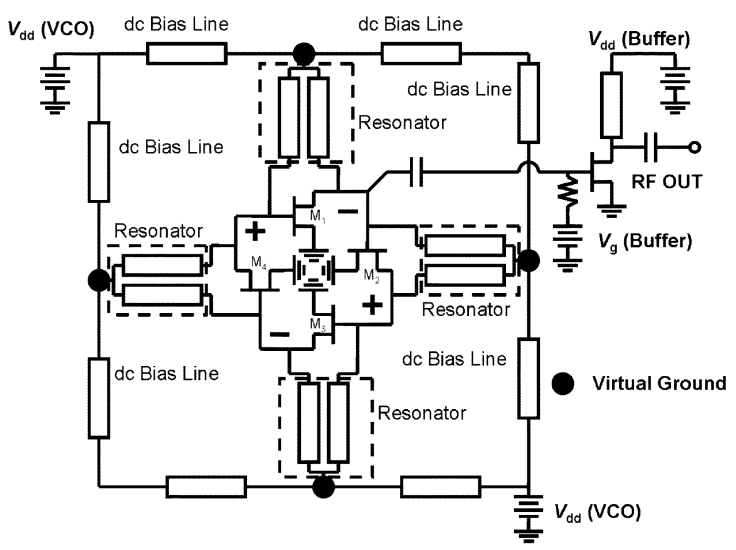

Fig. 4. Circuit schematic of the realized VCO using ring-coupled quad.

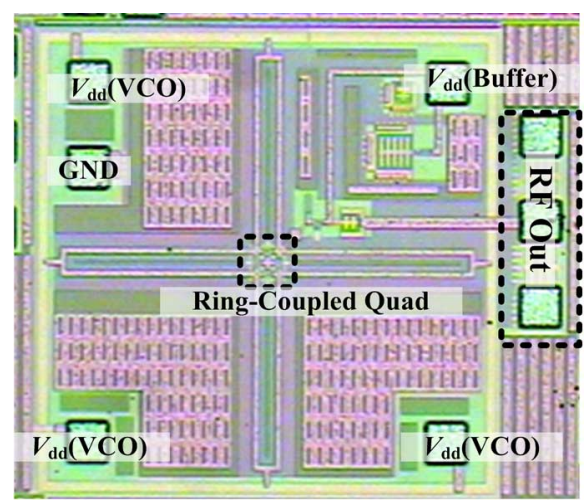

Fig. 5. Chip photo of the realized VCO using ring-coupled quad with a chip size of $620 \mu \mathrm{m} \times 550 \mu \mathrm{m}$.

Fig. 1(a) $\left(v_{d 2} / v_{g 1}\right)$ and (b) $\left(v_{d 4} / v_{g 1}\right)$ with the same device size (four-fingers and $16-\mu \mathrm{m}$ gate width) and equal resonators. All the parameters and coupling effects are considered in the simulation via full-wave EM solver [9]. The simulated results show that the ring-coupled VCO has higher loop gain than the cross-coupled VCO. Fig. 4 illustrates the complete circuit schematic of the VCO. Four transistors are connected as a ring, while the gate and drain of each transistor are connected to a resonator separately. Because the voltage of the drain and gate of each device are differential, each end of the resonators is virtual short. Therefore, the dc bias lines are connected to the end of the resonators without influencing the radio frequency (RF) performance of VCOs. The output voltage is sampled 


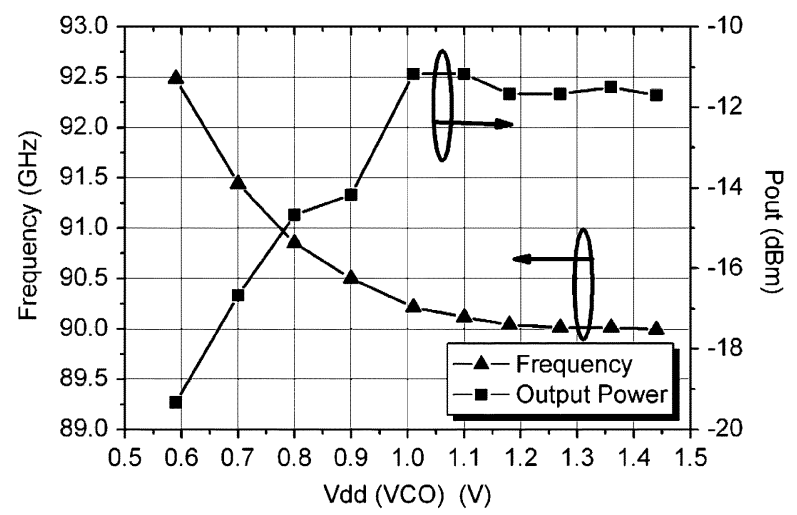

Fig. 6. Oscillation frequency and output power versus tuning voltage.

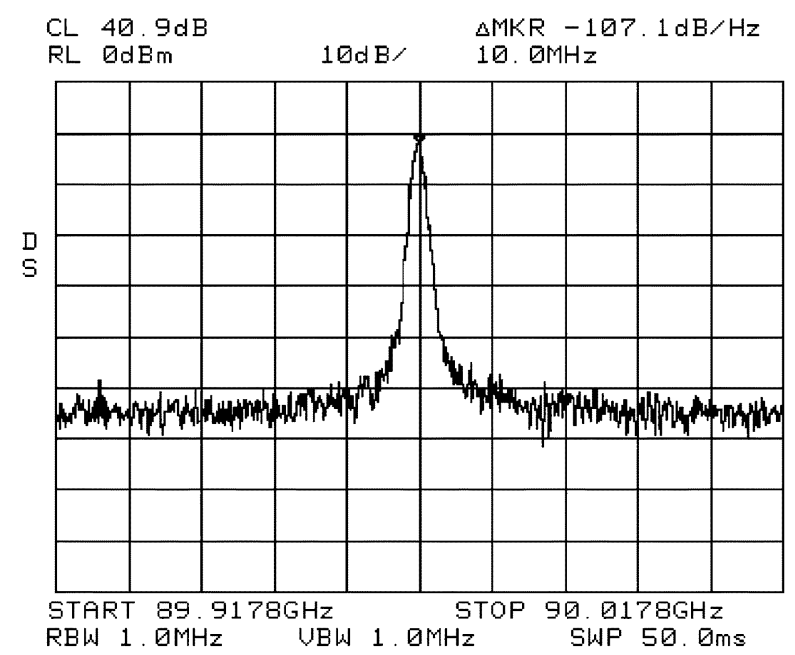

Fig. 7. Output spectrum of the VCO.

by a $0.05-\mathrm{pF}$ capacitor from the drain of $M_{1}$. The sampled voltage is amplified by a common source buffer amplifier. The frequency is tuned by the $V_{\mathrm{dd}}$ of the $\mathrm{VCO}$ [ $\left.V_{\mathrm{dd}}(\mathrm{VCO})\right]$. To fix the bias condition of the buffer amplifier, the 0.05 -pF capacitor blocks the dc level of the $V_{\mathrm{dd}}$ of the $\mathrm{VCO}$ and the gate voltage of the buffer amplifier. The $V_{\mathrm{dd}}$ of the $\mathrm{VCO}\left[V_{\mathrm{dd}}(\mathrm{VCO})\right]$ and the buffer amplifier $\left[V_{\mathrm{dd}}\right.$ (Buffer)] are fed seperately. Fig. 5 is the chip photo of the realized VCO with a chip size of $620 \mu \mathrm{m} \times 550 \mu \mathrm{m}$. The area of the cross-coupled quad is only $33 \mu \mathrm{m} \times 33 \mu \mathrm{m}$.

\section{Measurement Results}

The circuit was measured via on-wafer probing. The RF output pads of the chip were connected to the WR-10 waveguide by a W-band RF ground-signal-ground (GSG) probe. The loss of the waveguide and the probe is estimated to be $2 \mathrm{~dB}$. The output signal of VCO was down-converted to IF by the harmonic mixer HP11970W and measured using spectrum analyzer $8565 \mathrm{EC}$.

The drain voltage of the VCO is tuned from 0.6 to $1.45 \mathrm{~V}$ for frequency tuning. The drain and gate voltages of the buffer amplifier are both $0.8 \mathrm{~V}$ and the gate voltage of the buffer amplifier is $1.1 \mathrm{~V}$. Fig. 6 shows the oscillation frequency and output
TABLE I

COMPARISON OF THE REPORTED FUNDAMENTAL CMOS VCO OVER $90 \mathrm{GHZ}$ OPERATION FREQUENCY

\begin{tabular}{cccccc}
\hline \hline Ref. & Process & $\begin{array}{c}\text { Freq. } \\
(\mathrm{GHz})\end{array}$ & $\begin{array}{c}\mathrm{P}_{\text {out }} \\
(\mathrm{dBm})\end{array}$ & $\begin{array}{c}\text { Phase Noise } \\
(\mathrm{dBc} / \mathrm{Hz} @ \\
10 \mathrm{MHz} \\
\text { offset })\end{array}$ & $\begin{array}{c}\mathrm{P}_{\mathrm{DC}} \\
(\mathrm{mW})\end{array}$ \\
\hline$[5]$ & $90 \mathrm{~nm}$ & 103.9 & -65 & 110 & 30 \\
{$[6]$} & $130 \mathrm{~nm}$ & $88.5 \sim 90.1$ & $-14 \sim-18$ & $104 \sim 106$ & $15.75 \sim 7.5$ \\
$\begin{array}{c}\text { This } \\
\text { Work }\end{array}$ & $90 \mathrm{~nm}$ & $90 \sim 92.5$ & $-12 \sim-20$ & $\begin{array}{c}107.1 \\
(90 \mathrm{GHz})\end{array}$ & $87.2 \sim 14$ \\
\hline \hline
\end{tabular}

power versus drain voltage of the oscillator $\left[V_{\mathrm{dd}}(\mathrm{VCO})\right]$ in Fig. 4. The frequency can be tuned from 92.5 to $90 \mathrm{GHz}$ which gives $2.5-\mathrm{GHz}$ tuning range. The output power is higher than $-20 \mathrm{dBm}$ over the entire turning frequency. Fig. 7 illustrates the measured output spectrum when tuning voltage is $1.18 \mathrm{~V}$. The phase noise at $10-\mathrm{MHz}$ offset is $-107.1 \mathrm{dBc} / \mathrm{Hz}$. Table I lists the reported fundamental VCO over $90-\mathrm{GHz}$ operation frequencies. Although the over $100-\mathrm{GHz}$ operation frequency was demonstrated in [5], the output power is very low. Compared with [6], this work presents comparable output power and wide tuning range performance.

\section{CONCLUSION}

A new circuit topology of ring-coupled quad is proposed. It can provide a higher open loop voltage gain than the conventional crossed-coupled pair and the symmetrical layout can be achieved via this design approach. A 90-GHz VCO with 90-nm CMOS process is implemented with the proposed ring-coupled quad. The tuning range is $2.5 \mathrm{GHz}$ and the output power is higher than $-20 \mathrm{dBm}$. The experimental results reveal that this ring-coupled quad is suitable for high frequency VCO design.

\section{REFERENCES}

[1] Y.-H. Cho, M.-D. Tsai, H.-Y. Chang, C.-S. Lin, M.-F. Lei, H. Wang, C.-Y. Su, and C.-L. Chang, "A low phase noise 52-GHz push-push VCO in $0.18 \mu \mathrm{m}$ bulk CMOS technologies," in IEEE RFIC Symp. Dig., Jun. 2005, pp. 131-134.

[2] P.-C. Huang, R.-C. Liu, H.-Y. Chan, C.-S. Lin, M.-F. Lei, H. Wang, C.-Y. Su, and C.-L. Chang, "A $131 \mathrm{GHz}$ push-push VCO in $90 \mathrm{~nm}$ CMOS technology," in IEEE RFIC Symp. Dig., Los Angeles, CA, Jun. 2005, pp. 131-134.

[3] P.-C. Huang, M.-D. Tsai, H. Wang, C.-H. Chen, and C.-S. Chang, "A $114 \mathrm{GHz}$ VCO in $0.13 \mu \mathrm{m}$ CMOS technology," in IEEE ISSCC. Dig., San Francisco, CA, Feb. 2005, pp. 404-406.

[4] R.-C. Liu, H.-Y. Chang, C.-H. Wang, and H. Wang, "A $63 \mathrm{GHz}$ VCO using a standard $0.25 \mu \mathrm{m}$ CMOS process," in IEEE ISSCC. Dig., San Francisco, CA, Feb. 2004, pp. 446-447.

[5] L. M. Franca-Neto, R. E. Bishop, and B. A. Bloechel, "64 GHz and $100 \mathrm{GHz}$ VCOs in $90 \mathrm{~nm}$ CMOS using optimum pumping method," in IEEE ISSCC. Dig., San Francisco, CA, Feb. 2004, pp. 444-538.

[6] C. Cao and K. K. O, "A 90-GHz voltage-controlled oscillator with a 2.2-GHz tuning range in a 130-nm CMOS technology," in VLSI Symp. Dig., Kyoto, Japan, Jun. 2005, pp. 242-243.

[7] D. Nayak, L.-T. Hwang, and I. Turlik, "Simulation and design of lossy transmission lines in a thin-film multichip package," IEEE Trans. Comp., Packag., Manufact. Technol. A, vol. 13, no. 2, pp. 294-302, Jun. 1990.

[8] D. M. Pozar, "Microwave filters," in Microwave Engineering, 2nd ed. New York: Wiley, 1998, ch. 8, sec. 8.2, p. 431.

[9] “Sonnet User's Manual,” Sonnet Software, Inc., Liverpool, NY, 1998. 